\title{
INVOLUCRAR AL ALUMNADO EN LA EVALUACIÓN COMPARTIDA EN LA UNIVERSIDAD
}

Involve the students in the Shared Assessment in the University

Envolver os alunos na Avaliação Compartilhada na Universidade

\begin{abstract}
Este estudio se ha llevado a cabo dentro del proyecto de I+D+i: "Las competencias docentes en la formación inicial del profesorado de educación física”. Convocatoria de noviembre de 2013 del Programa Estatal de Investigación, Desarrollo e Innovación Orientada a los Retos de la Sociedad, en el marco del Plan Estatal de Investigación Científica y Técnica y de Innovación 2013-2016. Referencia: EDU 2013-42024-R. Duración: 3 años (2014-2016).
\end{abstract}

\section{Francisco Javier Castejón Oliva (1)}

Oidui Usabiaga Arruabarrena (2)

(1) Universidad Autónoma de Madrid (UAM), España. Teléfono: +34 914976681. Correo electrónico: javier.castejon@uam.es

(2) Universidad del País Vasco (UPV/EHU), España. Teléfono: +34 945013539. Correo electrónico: oidui.usabiaga@ehu.eus

\section{Resumen}

El objetivo del presente estudio fue comprobar los efectos percibidos por alumnado de dos universidades con respecto al compromiso adquirido mediante un contrato de aprendizaje. Se recogieron 85 cartas escritas por estudiantes universitarios y después de realizar un análisis inductivo, emergieron diversas categorías asociadas a la formación como profesionales, el esfuerzo dedicado, la evaluación y la actitud del profesorado. Los resultados muestran que el alumnado valora positivamente el contrato como forma de comprometerse con su proceso de enseñanza-aprendizaje, aunque también subrayan algunos aspectos que influyen en su implicación, como la alta inversión de tiempo de estudio, la cantidad de trabajo o la exigencia de estar al día.

Palabras clave: Evaluación; evaluación compartida; contrato de aprendizaje; aprendizaje profundo 


\begin{abstract}
The objective of the present study was to test the effects of a learning contract to improve the engagement of students in classes, and how that engagement is perceived. 85 letters written by undergraduates were collected and after an inductive analysis, emerged several categories associated with teacher training as a professional, dedicated effort, assessment, and teaching attitude. The results show that students appreciate the learning contract to engage with their teaching-learning process, although they also underline some aspects influencing your involvement, as the high investment of time of study, the amount of work or the requirement of being up to date.
\end{abstract}

Keywords: Assessment; shared evaluation; learning contract; deep learning

\title{
Resumo
}

O objetivo deste estudo foi testar os efeitos percebidos pelos estudantes de duas universidades sobre o compromisso assumido por um contrato de aprendizagem.85 cartas escritas por estudantes universitários foram coletadas e após a realização de uma análise indutiva surgiram várias categorias associadas a formação como profissional, esforço dedicado, avaliação e atitude dos professores.Os resultados mostram que os estudantes são positivos sobre o acordo como uma forma de se envolver com o processo de ensino e aprendizagem, mas também destacar alguns aspectos que influenciam o seu envolvimento, como o alto investimento de tempo de estudo, a quantidade de trabalho ou a exigência para manter-se atualizado.

Palavras-chave: Avaliação; avaliação compartilhada; contrato de aprendizagem; aprendizagem profunda

\section{Introducción}

La enseñanza alineada que plantea Biggs (2005, también Biggs \& Tang, 2007) supone una relación desde los objetivos hasta la evaluación y todo el proceso de enseñanza aprendizaje. Involucrar al alumnado en todo este proceso, de manera que pase desde un aprendizaje superficial a un aprendizaje profundo (Enwistle, 1989; Entwistle \& Peterson, 2004) es un reto. Hay diversos pasos que pueden darse con ese fin. Uno de ellos es implicar al alumnado con los contratos de aprendizaje (Brubaker, 2010; Przesmycki, 2000). 
Para comprobar si se cumplen los compromisos es necesario establecer elementos y factores reguladores del proceso de enseñanza aprendizaje, que informan sobre cómo se está desarrollando la materia. De entre las diversas posibilidades de actuación, Biggs (2005) señala la “carta a un amigo” como una buena estrategia para saber qué se está haciendo hasta el momento y cómo se puede regular el proceso de enseñanza aprendizaje para el profesorado y alumnado.

El objetivo es comprobar qué supone para el alumnado dar el paso de compromiso a participar de forma activa (contrato de aprendizaje) y cómo manifiesta esa participación cuando se ha avanzado en el desarrollo del programa de la asignatura, con la intención, también, de indicar al profesorado qué regulación puede hacerse respecto a ese avance.

\section{Método}

Mediante un diseño inductivo tratamos de comprobar qué manifiesta el alumnado sobre su implicación en la evaluación y para qué utilizamos esa información.

Ha participado alumnado de la Universidad del País Vasco (UPV/EHU) y la Universidad Autónoma de Madrid (UAM). De la UPV/EHU 65 cartas de alumnos (sobre 98 matriculados), de primer curso de la asignatura obligatoria de Fundamentos de pelota vasca y tenis del Grado en Ciencias de la Actividad Física y del Deporte. De la UAM han escrito 20 estudiantes (de los 30 matriculados) de Magisterio Educación Primaria, itinerario de EF, asignatura Iniciación deportiva.

Todos tenían firmado el contrato inicial que se les pide para ser evaluados por evaluación continua. Incluye un compromiso entre el docente y estudiantes sobre cómo proceder a lo largo de la asignatura. Eligen la calificación y según sea esa calificación tienen más o menos exigencia en lo que tienen que realizar adaptado el crédito ECTS.

Cuando se lleva la mitad del curso, un día de clase y al final de la sesión se les pide que escriban al amigo ficticio. La longitud máxima es una hoja por las dos caras y es anónimo. Un estudiante es el encargado de recoger esta carta, que se entrega al profesor. El profesor, una vez leídas, se compromete a contestar una carta común que entrega a cada uno y cada una. Como es normal, la carta del profesor es una síntesis de todas las aportaciones que hace el alumnado, pues el anonimato impide saber quién ha escrito lo que ha escrito. 
Se ha realizado un análisis documental de lo que han escrito los y las estudiantes. Siguiendo una metodología de análisis de contenido (Krippendorff, 1997; Rapley, 2014) se ha leído el documento hasta tres veces y se ha ido subrayando aquellos comentarios que se encuentran entre los más recurrentes y que suponen una manifestación cualitativa de lo que se hace en clase.

Los resultados (Tabla 1) señalan un conjunto de categorías que emergen del análisis documental y que nos ayudan a comprender qué señala el alumnado con el compromiso adquirido y su repercusión en el desarrollo de la asignatura.

Tabla 1.

Categorías emergentes del análisis realizado

\begin{tabular}{ll}
\hline \multicolumn{1}{c}{ Acuerdos de participación } & \multicolumn{1}{c}{$\begin{array}{c}\text { Conformidad con el hecho de que se haya firmado un } \\
\text { contrato y se respete }\end{array}$} \\
\hline Clase práctica & $\begin{array}{l}\text { Necesidad de ver en la práctica lo tratado en la teoría para cuando } \\
\text { sean docentes }\end{array}$ \\
\hline Analizar lo que se hace & $\begin{array}{l}\text { Entender los factores que permiten que lo que se trata en clase } \\
\text { tiene una aplicación en su futuro docente }\end{array}$ \\
\hline Aprender a ser docente & $\begin{array}{l}\text { Entender que la docencia requiere un conjunto de capacidades de } \\
\text { saber y saber hacer }\end{array}$ \\
\hline Mucho trabajo & $\begin{array}{l}\text { Necesidad de llevar la asignatura al día para evitar que no se } \\
\text { pueda seguir lo que se trata en las clases }\end{array}$ \\
\hline Evaluación formativa y & $\begin{array}{l}\text { Entender que la evaluación propuesta por el profesor (incluido el } \\
\text { contrato) está centrada en el aprendizaje y hace al estudiante } \\
\text { partícipe de su proceso }\end{array}$ \\
\hline Actuación del profesor & $\begin{array}{l}\text { Motivación, cercanía, disposición para ayudar al alumnado, } \\
\text { pretende que los estudiantes aprendan }\end{array}$ \\
\hline
\end{tabular}

\section{Resultados y discusión}

El alumnado de las dos universidades valora positivamente que exista un acuerdo previo, negociado con el profesorado, lo cual supone un compromiso asumido por las dos partes, que les lleva a centrarse en los aprendizajes a conseguir. Se aprecia que, al implicar al alumnado con los contratos de aprendizaje, se consigue pasar de un aprendizaje superficial a uno más profundo, de acuerdo con Enwistle (1989) y Entwistle y Peterson (2004). En este sentido, las propuestas del profesorado implicado son consideradas interesantes y efectivas de cara a su formación como profesionales de la actividad física y del deporte. La experiencia vivida la asocian con la posibilidad de aplicarlo en su futuro profesional.

Sin embargo, el alumnado cree que invierte un gran número de horas en estas asignaturas, donde sienten que no están habituados y que supone un cambio en su forma 
de proceder. Lo cual no es impedimento para percibir que la evaluación propuesta estaba centrada en su aprendizaje. Este sentimiento no hace más que reforzar que la evaluación pertenece a todos y a todos beneficia (Santos, 2000). En esta línea, han manifestado la importancia de sentir al profesor como una persona que "les engancha”- un “artista”-, pero que a su vez cumple con los rasgos de un "profesor serio” — que razona, argumenta y es justo-.

\section{Conclusiones}

El alumnado reivindica esta forma de proceder, valoran positivamente el contrato como forma de involucrarse, pero aparecen aspectos que puede que no se hayan tenido en cuenta en su momento y que repercuten en su implicación, como es el tiempo que tienen que dedicar al estudio, la cantidad de trabajo, la obligatoriedad de estar al día.

\section{Referencias}

Biggs, J. (2005). Calidad del aprendizaje universitario. Madrid: Narcea.

Biggs, J., \& Tang, C. (2007). Teaching for Quality Learning at University. What the Student Does (3 ed.). Berkshire: McGraw-Hill. SRHE and Open University Press.

Brubaker, N. D. (2010). Negotiating Authority by Designing Individualized Grading Contracts. Studying Teacher Education, 6(3), 257-267.

Entwistle, N. (1989). La comprensión del aprendizaje en el aula. Madrid: Paidós/MEC.

Entwistle, N. J., \& Peterson, E. R. (2004). Conceptions of learning and knowledge in higher education: Relationships with study behaviour and influences of learning environments. International Journal of EducationalResearch, 41, 407-428.

Krippendorff, K. (1997). Metodología de análisis de contenido: teoría y práctica. Barcelona: Paidós.

Przesmycki, H. (2000). La pedagogía de contrato. El contrato didáctico en la educación. Barcelona: Graó.

Rapley, T. (2014). Los análisis de la conversación, del discurso y de documentos en Investigación Cualitativa. Madrid: Morata.

Santos, M. A. (2000). La escuela que aprende. Madrid: Morata. 